SULlA NATURA DELLA FORZA PER GUI I CORPI SONO RESPINTI da poli di una galamita. - Lezione del Prop. TYNDall. all' Istituzione Reale della Gran-Brelagna nell' Adunanza delli 26 Gemnajo 185\%5. (Estralto dell' Autore).

Il Prof. Tyndall comincia dal mostrare che i corpi sono respinti dai poli di una calamita in virtù di uno stato di eccitamento in essi indotto dalla calamita stessa. La repulsione del bismuto e l'attrazione del ferro dolce seguono precisamente le stesse leggi allorquando la forza della calamita varia, essendo esse proporzionali non semplicemente alla forza della calamita, ma: dentro certi limiti, al quadrato di quella forza. Per il caso del ferro si spiega questa legge dicendo che sotto l'influenza magnetica il ferro è convertito in una vera calamita temporaria, di cui la forza varia col magnetisino inducente; nel caso del bismuto il resultato può solamente essere spiegato ammettendo che la massa diamaguetica è convertita in un vero diamagnete.

E stato pure mostrato che la condizione eccitala da uno dei poli non è la stessa di quella eccitata da un polo contrario.

Se la condizione eccitata fosse indipendente dalla qualità del polo, ne seguirebbe che $i$ due poli di nome contrario agendo simultaneamente sul bismuto, dovrebbero respingerlo, lo che non è. Due poli dello stesso nome producono repulsione; ma quando sono di nome contrario e di forza eguale, la condizione eccitata in uno dei poli è neutralizzata dall' altro, e non vi è repulsione. L' Autore ha operato sopra corpi magnetici e diamagnetici con tutte le forze capaci di un' azione magnetica, cioè $1 .^{\circ}$ colla calamita sola, $20^{\circ}$ colla corrente elettrica, $3 .^{0}$ colla calamita e colla corrente combinate insicme. In queste ricerche è necessario di attendere alla struttura dei corpi, e forse l'aver trascurata questa circostanza ha introdotto molti errori in questa parte della scienza. L'Autore ha preparato una massa compatta di bismuto comprimendo con una pressa idraulica in uno stampo di metallo della polvere di bismuto puro. La massa 
cosi ottenuta sospesa nel campo magnelico, essendo orizzontale alla linea di compressione, si comporta come un corpo magnetico, cioé colla dinensione più lunga da polo a polo, fissandosi quella linea normale alla linea polare. Questa identitì fra gli effetti del bismuto compresso e le sostanze magnetiche ordinarie si verificava usando la corrente elettrica, e la corrente e la calamita combinate.

Nella stessa guisa comprimendo una polvere magnetica, si ottengono sbarre che si comportano come se fossero sbarre di corpi magnetici. Chiamando quelle sbarre che mostrano l'azione inagnetica o dianagnetica ordinaria sbarre normali e anormali quelle compresse, ne segue che una sbarra anormale di una classe di corpi si comporta precisanente come una sbarra normale dell'altra classe; ma quando paragoniamo insieme sbarre normali o anormali, dell una e dell' altra classe, l'antitesi dell' azione è perfetta. L' esperienza dimostra che se ciò che Gauss chiama distribuzione ideale del magnelismo nelle sbarre magnetiche fosse invertita, si avrebbe una distribuzione che produrrebbe tutti gli effetti dei corpi diamagnetici.

I' Autore si è specialmente occupato della polarita diamagnetica. Una spirale schiacciata, di cui il diamotro interno era 1 pollice e l'esterno 7 pollici e lunga 1 pollice, era fissata solidamente sopra una tavola essendo verticale il pi:tno delle spire. Una sbarra di bismuto lunga 5 pollici e di 0,4 di pollice di diametro era sospesa per il suo centro di gravità in modo da poter liberamente oscillare dentro la spirale che la circondava. Così disposto l'apparecchio, l'Autore ha fatto le seguenti esperienze. Fatta passare una corrente di 20 pile di Grove attraverso la spirale che circonda la sbarra di bismuto nel tempo che si rendeva attiva un' elettro-calamita di cui l'asse incontrava perpendicolarmente una delle estremità della sbarra, si vedeva la sbarra stessa ora attratta ora respinta dal polo, secondo il nome del polo, e secondo la direzione della corrente della spirale. Questi movimenti erano accresciuti e all'incirca raddoppiati allorchè si faceva agire sull' altra estremitit della sbarra di bismuto e dalla parte opposta il polo contrario di un' altra 
calamita, nel qual caso i due poli agivano come una coppia meccanica. Se i due poli elano dello stesso nome non vi era più alcun movimento nella sbarra, mentre acquistavano maggior prontezza e decisione usando quattro elettro-calamite, cioè, interponendo ognuna delle estremiti della sbarra fra due poli di nome contrario. In tulti $i$ casi $i$ movimenti erano opposti a quelli oltenuli colla sbas'ra di bismuso, sostituendo a questa una sbarra di ferro.

La questione della polarita diamagnetica fu in seguito sottoposta ad un' altra prova che cadeva sotto il dominio di alcuni principj di meccanica. S'imagini di avere una superficie polare molto estesa, onde ottenere un campo di forza magnetica uniforme, e si sospenda dinanzi a questa superficie una leva, e, posta ad una estremita o fissata in traverso alla lunghezza della leva, una sbarra normale di bismuto: un peso dalla parte opposta fa equilibrio alla sbarra di bismuto, e il sistema essendo sospeso per il suo centro di gravita, la leva e la sbarra possono oscillare orizzontalmente. Supponiamo che la leva sia inclinata di circa $45^{\circ}$ coll asse magnetico; se la forza agente sopra il bismuto fosse purcmente repulsiva, cioè se lo stato diamagnetico fosse unipolare, ogni particella della massa di bismuto tenderebbe ad allontanarsi dal polo. Invece l' esperienza dimostra che mettendo in azione l'elettro-calamita si ottiene un moto contrario, ciod la leva si avvicina al polo. L'A. considera questo fatto come spiegato dall' ipotesi della polarita diamagnetica. In questa ipotesi le estremità della sbarra di bismuto hanno poli contrari, uno dei quali è attratto e l'altro respinto dai poli della calamita; ma la forza agente sopra il polo attrattivo e applicata ad una maggiore distanza dall asse di sospensione di quella che agisce sul polo ripulsivo, e siccome la disposizione è tale che le intensiti assolute delle forze agenti sopra i due poli differiscono pochissimo l'una dall'altra, l'estremiti piu lontana attratta si muove verso la superficie polare perchè soggetta a un momento più grande di rotazione.

L'A. appoggia questa conclusione con una esperienza, tentata sostituendo alla sbarra di bismuto un ago calamitato soggetto all azione magnetica della terra.

Vol. II. 
66

Una memoria presentata dall A. alla Socicti Reale di Londra nel Novembre decorso, e di cui una parte forma il soggetto della lezione Bakeriana dell'anno presente, contiene una più estesa discussione di questo soggetto, colla quale l'A. spiega in un modo, che egli crede soddisfacente, le difficoltà addotte dal Matteucci nel suo Cours special contro la spiegazione data dall' Autore dei movimenti del bismuto cristallizzato o compresso nel campo magnetico.

SOPRA UNa NUOVA CLASSE DI RADICALI ORgayici- $-\Lambda$. WUR'TZ.

Compl. rend. de l'Acad. des Sciences, $\iota$. XL. p. 128\%.

\section{( Estrallo)}

$\dot{E}$ noto che -Kolbe e Frankland hanno isolato $\mathrm{i}$ gruppi organici di cui i Chimici ammettono l'esistenza negli alcoli e negli eteri.

I due Chimici precitati sottoponendo alla elettrolisi gli acidi grassi volatili $\mathrm{C}^{n} \mathrm{H}^{\mathrm{n}} \mathrm{O}^{4}$, o decomponendo collo zinco gli eteri idrojodici, hanno ottenuto una serie d'idrogeni carbonati che rappresentano colle formule seguenti:

\section{$\mathrm{C}^{2} \mathrm{H}^{3} \quad$ Metile \\ $\mathrm{C}^{6} \mathrm{H}^{5}$ Etile, \\ $\mathrm{C}^{8} \mathrm{H}^{9}$ Butile \\ $\mathrm{C}^{40} \mathrm{H}^{41}$ Amile}

In seguito è stato aggiunto a questa seric il caproile $\mathrm{C}^{12} \mathrm{H}^{13}$.

Secondo le idee di Kolbe e Frankland, i radicali alcolici avrebbero allo stato libero lo stesso peso atomico e la stessa forma molecolare che hanno nelle loro combinazioni.

Essi esistercbbero negli alcoli quali sono allo stato libero, ed in quest'ultima condizione il loro equivalente corrisponderebbe a due volumi di vapore. Per altro questo modo di vedere non $\grave{e}$ stato adotlato da tutti i Chimici. 